\title{
Noncoding RNA danger motifs bridge innate and adaptive immunity and are potent adjuvants for vaccination
}

\author{
Lilin Wang, Dan Smith, Simona Bot, Luis Dellamary, Amy Bloom, and Adrian Bot \\ Department of Immunology, Astral Inc. of Alliance Pharmaceutical Corporation, San Diego, California, USA
}

\begin{abstract}
The adaptive immune response is triggered by recognition of $\mathrm{T}$ and $\mathrm{B}$ cell epitopes and is influenced by "danger" motifs that act via innate immune receptors. This study shows that motifs associated with noncoding RNA are essential features in the immune response reminiscent of viral infection, mediating rapid induction of proinflammatory chemokine expression, recruitment and activation of antigen-presenting cells, modulation of regulatory cytokines, subsequent differentiation of $\mathrm{Th} 1 \mathrm{cells}$, isotype switching, and stimulation of cross-priming. The heterogeneity of RNA-associated motifs results in differential binding to cellular receptors, and specifically impacts the immune profile. Naturally occurring double-stranded RNA (dsRNA) triggered activation of dendritic cells and enhancement of specific immunity, similar to selected synthetic dsRNA motifs. Based on the ability of specific RNA motifs to block tolerance induction and effectively organize the immune defense during viral infection, we conclude that such RNA species are potent danger motifs. We also demonstrate the feasibility of using selected RNA motifs as adjuvants in the context of novel aerosol carriers for optimizing the immune response to subunit vaccines. In conclusion, RNA-associated motifs produced during viral infection bridge the early response with the late adaptive phase, regulating the activation and differentiation of antigen-specific $B$ and $T$ cells, in addition to a short-term impact on innate immunity.
\end{abstract}

J. Clin. Invest. 110:1175-1184 (2002). doi:10.1172/JCI200215536.

\section{Introduction}

During viral infection, specific $\mathrm{T}$ lymphocytes are exposed to foreign epitopes displayed by MHC molecules (1), and the B lymphocytes recognize antigens in soluble form (2). Proliferation and differentiation of lymphocytes defines the adaptive immune response carried out by specific effector and memory cells. During the initial phase of the immune response, the innate immune system recognizes microbe-associated motifs as well as lesion-triggered endogenous danger signals that direct the subsequent differentiation of specific lymphocytes and the overall profile of the immune response (3). In the absence of danger signals, the $\mathrm{T}$ and $\mathrm{B}$ cell responses are reduced in magnitude and immune tolerance may result, particularly at moderate to high doses of antigen (4). It has

Received for publication March 27, 2002, and accepted in revised form August 16, 2002.

Address correspondence to: Adrian Bot, Department of Immunology, Alliance Pharmaceutical Corporation,

3040 Science Park Road, San Diego, California 92121, USA.

Phone: (858) 410-5259; Fax: (858) 410-5612;

E-mail:axb@allp.com.

Conflict of interest: All authors are full-time employees of Alliance Pharmaceutical Corporation and enrolled in a stock option program.

Nonstandard abbreviations used: double-stranded RNA (dsRNA); Toll-like receptor (TLR); polyA:polyU (pA:pU); polyI:polyC (pI:pC); dendritic cell (DC); antigen-presenting cell (APC); T cytotoxic 1 cells (Tc1); ovalbumin (OVA); cholera toxin subunit B (CTB); human IgG (hIgG); ultraviolet (UV); MadinDarby canine kidney (MDCK); short-chain phospholipid vector (SC-V); UV-inactivated WSN virus (UV-WSN); 50\% tissue culture infective dose $\left(\mathrm{TCID}_{50}\right)$; magnetic cell sorting (MACS);

phycoerythrin (PE); single-stranded RNA (ssRNA); spot-forming colony (SFC). recently been proposed that this is a critical mechanism in discriminating between innocuous and "dangerous" antigens associated with infection (3). This also sheds a different light on the strategy of the immune system to discriminate between self and nonself, previously thought to be determined exclusively at the level of the antigen-receptor repertoire (5).

A significant number of viral infections are associated with, or result in production of, RNA species in the absence of infection. Such RNAs are either genomic fragments (in the case of viruses containing double-stranded RNAs, or dsRNAs), replicative intermediates, or stemand-loop structures (6) that are recognized by innate immune receptors such as Toll-like receptor 3 (TLR-3) (7) and trigger production of IFN type I and other soluble mediators (8). In addition, certain dsRNA motifs such as polyI:polyC (pI:pC) have been shown to activate immature dendritic cells (DCs) to a stage where they act as professional antigen-presenting cells (APCs) (9). Despite the fact that pI:pC and IFN type I were recently shown to influence the antibody response to a protein antigen (10), most of the information obtained about dsRNA immune modulatory motifs has resulted from models of innate immunity (11). Therefore it has not been clear whether motifs associated with double-stranded or other RNA species have only a limited effect on the adaptive immune response or act as potent danger signals that prevent immune tolerance and direct the differentiation of specific $\mathrm{T}$ cells. In addition, the critical question as to whether there is a multiplicity of RNA-associated danger motifs with potential differential impact on the immune response has not been addressed. Furthermore, it has not been determined whether noncoding RNA motifs can 
facilitate the induction of class I-restricted immune responses during viral infections, thought until recently to occur primarily as a result of abortive or productive infection of APCs (12).

In the current study, we demonstrate that in addition to the single- versus double-stranded nature of RNA, oligonucleotide composition is a critical determinant for recognition of noncoding RNA motifs by innate immune receptors. In addition, heterogeneous synthetic RNA motifs have a potent and differential impact on adaptive immunity, mediating the major features of immunity against viruses. Similarly, naturally occurring dsRNA induced both activation of DCs and enhancement of specific responses. Finally, we show that defined synthetic RNA motifs can be effectively used in the context of vaccination to trigger enhanced antibody, Th1, and T cytotoxic 1 cells (Tc1) responses.

\section{Methods}

Antigens and immunomodulators. A panel of 18 singlestranded and double-stranded synthetic RNAs (Table 1) was purchased from Sigma-Aldrich (St. Louis, Missouri, USA) and dissolved in sterile PBS. The RNAs were used as pools or individually. Low-endotoxin ovalbumin (OVA) was purchased from Sigma-Aldrich Cholera toxin subunit B (CTB) was purchased from Calbiochem-Novabiochem Corp. (San Diego, California, USA); CFA from Becton, Dickinson and Co. (Franklin Lakes, New Jersey, USA); and human IgG (hIgG) from Sigma-Aldrich. The recombinant gp 140 HIV antigen that retains conformational epitopes and has the ability to trimerize was derived from gp160 envelope protein of strain IIIB by introducing a stop mutation (13). The antigen was expressed by a vaccinia virus vector (generously provided by Bernard Moss, NIH, Bethesda, Maryland, USA) in BS-C-1 cells (American Type Culture Collection, Rockville, Maryland, USA) and was purified by lentil lectin Sepharose chromatography (Amersham Pharmacia Biotech, Piscataway, New Jersey, USA). The identity of gp140 antigen was confirmed by Western blot analysis using HIV envelope-specific antibodies purchased from Fitzgerald Industries International (Concord, Massachusetts, USA). Influenza virus (strain A/WSN/32 H1N1) was grown on Madin-Darby bovine kidney carcinoma cells and purified from supernatants by sucrose gradient centrifugation. For virus inactivation, the virions were exposed to short-wave ultraviolet (UV) light for $15 \mathrm{~min}$ utes under stirring. Inactivation was confirmed by virus titration on permissive Madin-Darby canine kidney (MDCK) carcinoma cells. Recombinant mouse $\operatorname{IgG}_{2} \mathrm{~b}$ bearing the I- $\mathrm{E}^{\mathrm{d}}$-restricted hemagglutinin-derived peptide SFERFEIFPKE within the variable region (IgHA) was obtained and purified as characterized previously (14). Animals. C57BL/6, BALB/c, and TLR4-/- $\mathrm{C} 3 \mathrm{H} / \mathrm{HeJ}$ female mice between 6 weeks and 8 weeks of age were purchased from The Jackson Laboratory (Bar Harbor, Maine, USA) and housed under specific pathogen-free conditions at Alliance Pharmaceutical Corp. according to institutional regulations. (Key observations in
$\mathrm{C} 57 \mathrm{BL} / 6$ and $\mathrm{BALB} / \mathrm{c}$ mice were reproduced in $\mathrm{C} 3 \mathrm{H} / \mathrm{HeJ}$ mice having deficient responsiveness to endotoxin.) Female Sprague-Dawley rats (250-330 g) were purchased from Taconic (Germantown, New York, USA) and were housed under similar conditions.

Engineering of the delivery vector. To construct aerosol vectors, phospholipids were homogenized in water (to form micelles) and mixed with the excipients and the actives. The water was then removed, resulting in a dry powder that could be administered as an aerosol. In a pilot experiment, various classes of biocompatible phospholipids were tested for compatibility with polynucleotide release from such complexes. Short-chain (C8) phospholipids were more effective than large phospholipids such as dipalmitoylphosphatidylcholine and thus were selected as the major lipid excipient for the tested aerosol vectors (short-chain phospholipid vectors, or SC-V). Such SCVs are compatible with mucosal or parenteral delivery. The antigen content was $10 \%$ (wt/wt), synthetic RNAs made up 5\% (wt/wt), and as minor excipient we used lactose (20\%). Control particles were loaded with antigen and CTB $(1 \% \mathrm{wt} / \mathrm{wt})$, with OVA only, or were devoid of antigen and immunomodulatory compounds. Before formulation, the immunologically active compounds were tested for endotoxin by Limulus amebocyte lysate assay (BioWhittaker Inc., Walkersville, Maryland, USA), and when necessary, LPS traces were eliminated by endotoxin-removal columns (Detoxi-Gel Endotoxin Removal Gel AffinityPak; Pierce Biotechnology Inc., Rockford, Illinois, USA). The physicochemical properties of aerosol vectors, revealed by scanning electron micrographs, were defined prior to use. The majority of the particulate structures displayed a quasispherical shape, with diameters between $1 \mu \mathrm{m}$ and $3 \mu \mathrm{m}$.

Immunization, challenge, and measurement of virus titers. Mice and rats were primed by intratracheal instillation or aerosolization using a PennCentury insufflator device (PennCentury Inc., Philadelphia, Pennsylvania, USA) according to the manufacturer's instructions. Mice received two viral boosts intranasally at 2 -week intervals. For induction of high-zone tolerance, the mice were primed by intravenous injection. Finally, for the induction of strong immune responses, mice were

\section{Table 1}

Nucleotide composition of synthetic RNA compounds used for screening of immunomodulatory motifs

\begin{tabular}{|c|c|c|}
\hline Category of RNA ${ }^{A}$ & & Nucleotide composition ${ }^{B}$ \\
\hline Single-stranded & $\begin{array}{l}\text { Pool } 1 \\
\text { Pool } 2 \\
\text { Pool } 3 \\
\text { Pool } 4\end{array}$ & $\begin{array}{c}\mathrm{p}(\mathrm{A}) ; \mathrm{p}(\mathrm{C}) ; \mathrm{p}(\mathrm{G}) ; \mathrm{p}(\mathrm{I}) ; \mathrm{p}(\mathrm{U}) \\
\mathrm{p}(\mathrm{G}, \mathrm{U}) ; \mathrm{p}(\mathrm{C}, \mathrm{U}) ; \mathrm{p}(\mathrm{A}, \mathrm{C}) ; \mathrm{p}(\mathrm{I}, \mathrm{U}) \\
\mathrm{p}(\mathrm{C}, \mathrm{I}) ; \mathrm{p}(\mathrm{A}, \mathrm{U}) ; \mathrm{p}(\mathrm{A}, \mathrm{G}) \\
\mathrm{p}(\mathrm{A}, \mathrm{C}, \mathrm{G}) ; \mathrm{p}(\mathrm{A}, \mathrm{C}, \mathrm{U}) ; \mathrm{p}(\mathrm{A}, \mathrm{G}, \mathrm{U})\end{array}$ \\
\hline Double-stranded & Pool 5 & pC:pG; pA:pU; pl:pC \\
\hline
\end{tabular}

ASynthetic RNAs were dissolved in sterile PBS and used individually or mixed in equivalent amounts as shown above. The total number of 18 compounds was initially divided for screening purposes in four pools obtained by mixing ssRNAs, and in a fifth pool of dsRNAs. The total concentration of RNA in each pool was $2 \mathrm{mg} / \mathrm{ml}$. ${ }^{\mathrm{P}} \mathrm{p}$, poly. 
immunized subcutaneously with antigen emulsified in CFA. The amounts of antigens used for priming, boosts, or induction of tolerance were: OVA, $100 \mu \mathrm{g}$; HIV gp140, $10 \mu \mathrm{g}$; hIgG, $200 \mu \mathrm{g}$; and sucrose-purified UV-inactivated WSN virus (UV-WSN), $20 \mu \mathrm{g}$. The amount of synthetic RNA used was $40-50 \mu \mathrm{g} /$ dose with or without antigen, incorporated or not into $\mathrm{SC}-\mathrm{V}$. The amount of CTB per dose was $10 \mu \mathrm{g}$. Nonformulated antigens were delivered in saline. Formulated antigens were delivered in perfluorocarbon, an inert vehicle that is compatible with the SC-V matrix (total volume of instillation or aerosolization, $40-45 \mu \mathrm{l}$ ).

For virus challenge, C57BL/ 6 and TLR4 $4^{-/-} \mathrm{C} 3 \mathrm{H} / \mathrm{HeJ}$ mice under Metofane anesthesia were infected with $10^{4}$ $\mathrm{TCID}_{50}$ ) of live WSN virus via the nasal route. On day 5 after infection, the mice were sacrificed and lungs were retrieved, homogenized, and stored at $-70^{\circ} \mathrm{C}$. Virus titers were measured by 48 -hour incubation of serial dilutions of samples with permissive MDCK cells, followed by standard hemagglutination with chicken red blood cells (Animal Technologies Inc., Tyler, Texas, USA). The endpoint titers were estimated in triplicate measurements by interpolation (15) and expressed as total $\mathrm{TCID}_{50}$ /organ.

Measurement of antibody and T cell response. Antibody responses were measured by ELISA. In brief, wells were coated with antigen $(2 \mu \mathrm{g} / \mathrm{ml} \mathrm{gp} 140,8 \mu \mathrm{g} / \mathrm{ml}$ sucrosepurified virus, $10 \mu \mathrm{g} / \mathrm{ml} \mathrm{hIgG}$, or $10 \mu \mathrm{g} / \mathrm{ml}$ OVA) and blocked with Sea Block (Pierce Biotechnology Inc.). Serial dilutions of serum and bronchoalveolar lavage fluid were incubated in the wells for at least 2 hours at room temperature. After washing the wells, the assay was developed with anti-mouse IgG antibody coupled with alkaline phosphatase (Sigma-Aldrich) followed by addition of substrate (pNPP; Sigma-Aldrich) and measurement with an automatic microtiter plate reader (THERMOmax; Molecular Devices Corp., Sunnyvale, California, USA) equipped with SoftMax software.

For measurement of cellular responses, splenic cell suspensions were obtained by passing spleenic tissue through 70- $\mu \mathrm{m}$ nylon Falcon strainers (Becton, Dickinson and Co.) followed by lysis of red blood cells with red blood cell lysis buffer (Sigma-Aldrich). The lymphocytes from the pulmonary lymphoid tissue were isolated by collagenase (Sigma-Aldrich) digestion of lung tissue followed by Ficoll-Paque (Amersham Pharmacia Biotech) gradient centrifugation. The $T$ cell response was measured by ELISpot analysis as follows: 96-well $45-\mu \mathrm{m}$ mixed cellulose ester plates (Millipore Corp., Bedford, Massachusetts, USA) were coated with $4 \mu \mathrm{g} / \mathrm{ml}$ of rat anti-mouse antibodies against IFN- $\gamma$, IL-2, or IL-4. (Pharmingen, San Diego, California, USA). After blocking with $10 \% \mathrm{FCS}$ in sterile saline for 1 hour at $37^{\circ} \mathrm{C}$, spleen cell suspensions were added at $5 \times 10^{5}$ cells per well, with or without antigens/peptides. In the case of pulmonary lymphocytes, effector cells were mixed 1:1 with mitomycin-treated splenic stimulator cells before stimulation. In some experiments, we used $\mathrm{CD}^{+} \mathrm{T}$ cells separated by magnetic cell sorting (MACS; Miltenyi Biotec, Auburn, California, USA). For stimulation, we used graded amounts of antigen (OVA, gp140, hIgG, or sucrose-purified WSN virus) or peptides: class II-restricted HA SFERFEIFPKE, or class I-restricted SIINFEKL or HIV V3-derived R10K peptide (16). Both the $\mathrm{H}-2 \mathrm{~K}^{\mathrm{b}}$-restricted OVA-derived SIINFEKL peptide and the $\mathrm{H}-2 \mathrm{~K}^{\mathrm{d}}$-restricted $\mathrm{V} 3$-derived peptide are wellknown epitopes recognized by MHC class I-restricted $\mathrm{CD}^{+} \mathrm{T}$ cells $(16,17)$. At 72 hours after stimulation, the assay was developed with biotinylated rat anti-mouse cytokine antibodies (Pharmingen) followed by streptavidin-conjugated horseradish peroxidase (BioSource International, Camarillo, California, USA) and insoluble AEC substrate. The results were measured using an automatic imaging system (Navitar Micromate; Navitar Inc., Rochester, New York, USA) equipped with multiparametric analysis software (Image-Pro; Media Cybernetics Inc., Silver Spring, Maryland, USA).

Measurement of chemokine gene expression. The level of chemokine expression in the lungs of mice treated 1 day previously with synthetic RNA or control was measured by DNA array technology as follows: total RNA was isolated from lungs using an RNeasy kit (QIAGEN Inc., Valencia, California, USA). RNAs were further purified by treatment with RNase-free DNase I (Stratagene, San Diego, California, USA). DNA array was performed using the Nonrad GEArray kit from SuperArray Bioscience Corp. (Bethesda, Maryland, USA). Briefly, cDNA probes were synthesized using Moloney murine leukemia virus reverse transcriptase with dNTP mix containing biotin16-dUTP. The GEArray membranes were prehybridized at $68^{\circ} \mathrm{C}$ for $1-2$ hours. Hybridization was carried out by incubation of the membranes with biotin-labeled cDNA. The hybridized membranes were washed twice in $2 \times$ SSC with $1 \%$ SDS and twice in $0.1 \times$ SSC with $0.5 \%$ SDS. The membranes were further incubated with alkaline phosphatase-conjugated streptavidin (BioSource International) and finally developed with CDP-Star chemiluminescent substrate (New England Biolabs Inc., Beverly, Massachusetts, USA). Signal intensity was measured with an Image-Pro analysis system equipped with Gel-Pro software (Media Cybernetics).

Flow cytometry. Cell suspensions from lungs of mice treated 1 day previously with $50 \mu \mathrm{g}$ of synthetic RNA or saline were prepared by collagenase digestion and Ficoll gradient centrifugation as above. The cells were resuspended in PBS containing $1 \%(\mathrm{vol} / \mathrm{vol})$ mouse serum (Sigma-Aldrich) and 1\% (wt/vol) BSA, fraction $\mathrm{V}$ (Sigma-Aldrich), and stained with phycoerythrinlabeled (PE-labeled) rat anti-mouse CD11b, PE-labeled hamster anti-mouse CD11c, or the appropriate PElabeled isotype control (all from Pharmingen) at a concentration of $1 \mu \mathrm{g}$ antibody $/ 10^{6}$ cells for 40 minutes on ice. The analysis was carried out on a FACSCalibur instrument (Becton, Dickinson and Co.). Nonviable cells were gated out using propidium iodide.

Magnetic separation and adoptive transfer. Professional APCs such as $\mathrm{CD} 11 \mathrm{c}^{+} \mathrm{DCs}$ were separated from spleens of $\mathrm{BALB} / \mathrm{c}$ mice using magnetic beads coupled to rat anti-mouse CD11c antibodies (Miltenyi Biotec). In brief, 
a

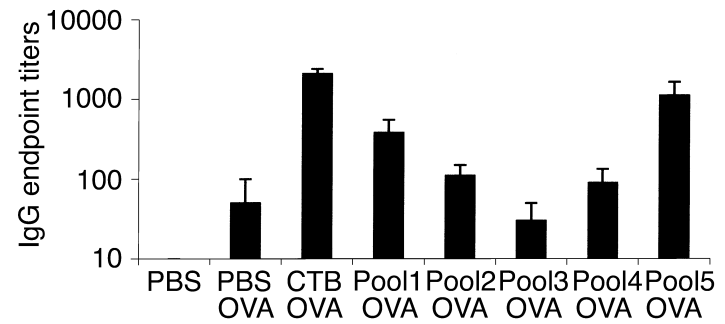

b

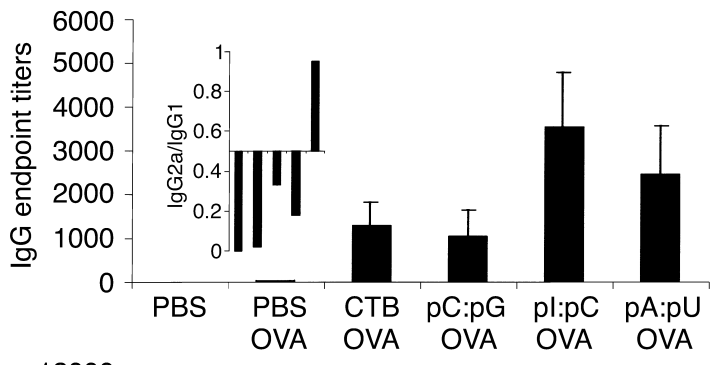

C

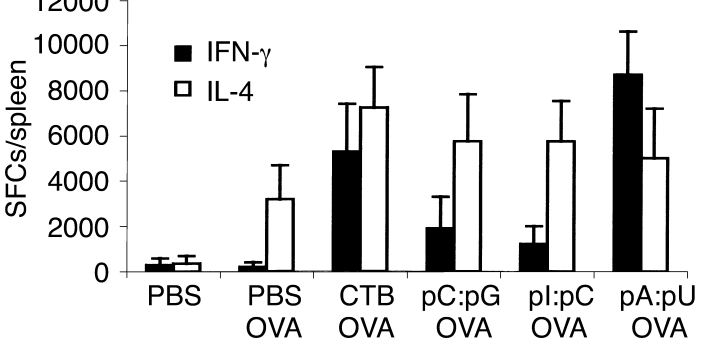

\section{Figure 1}

Effect of various synthetic RNA motifs on specific antibody and T cell immunity. (a) The impact of various RNA pools (see Table 1) on adaptive immunity was measured in C57BL/ 6 mice coimmunized with OVA via the respiratory tract. Mice were immunized intratracheally, followed by two boosts 2 weeks apart by intranasal instillation. The antibody response was expressed as mean \pm SEM of IgG endpoint titers ( $n=4$ /group). As controls, we used dose-matched OVA in sterile PBS, OVA with CTB, and PBS alone, respectively. (b) Effect of various dsRNA motifs on the induction of antibody response to OVA; results are expressed as in $\mathbf{a}$. The data are representative of two independent experiments. Inset: the ratio of mean $\operatorname{lgG} 2 \mathrm{a}$ and $\lg \mathrm{G} 1$ titers to OVA. The order from left to right is similar to that in the larger graph: PBS OVA, CTB OVA, pC:pG OVA, pl:pC OVA, and pA:pU OVA. (c) Magnitude and profile of $T$ cell response induced by OVA together with various dsRNA motifs in female C57BL/ 6 mice. The results obtained by ELISpot analysis were expressed as mean \pm SEM of the number of IFN- $\gamma$ and IL-4 spot-forming colonies (SFCs) per spleen ( $n=4$ /group). The results are representative of two independent experiments.

single-cell suspensions were resuspended in MACS buffer (BSA and EDTA) at $10^{7}$ cells $/ \mathrm{ml}$, incubated for 15 minutes on ice with magnetic beads, washed, and passed through magnetic columns. The columns were washed three times before elution, followed by two consecutive washings and in vitro overnight pulsing of cells with 100 $\mu \mathrm{g} / \mathrm{ml}$ of IgHA with or without $50 \mu \mathrm{g} / \mathrm{ml}$ of RNA motifs or $5 \mathrm{ng} / \mathrm{ml}$ recombinant IL-12 (BioSource International). Alternatively, the cells were incubated overnight with IgHA on wells previously coated with rat anti-mouse CD40 monoclonal antibody (Pharmingen). The cells were washed, resuspended in balanced sterile saline, and adoptively transferred by subcutaneous injection into naive $\mathrm{BALB} / \mathrm{c}$ mice $\left(2.5 \times 10^{5} \mathrm{APCs} /\right.$ mouse $)$. The $\mathrm{T}$ cell response was measured at 14 days by IL-2 ELISpot analysis after stimulation with HA class II-restricted peptide, as described above.

Untouched $\mathrm{CD}^{+} \mathrm{T}$ cells were separated from cell suspensions of spleens harvested from immunized mice using a $\mathrm{CD}^{+} \mathrm{T}$ cell isolation kit according to the manufacturer's instructions (Miltenyi Biotec).

Tagging of synthetic dsRNA and staining of APCs. Synthetic pA:pU dsRNA was tagged with fluorescent dye (Alexa Fluor 488) according to the manufacturer's instructions (Molecular Probes Inc., Eugene, Oregon, USA). In brief, following the removal of endotoxin as described above, pA:pU was labeled using the ULYSIS nucleic acid labeling system (Molecular Probes Inc.) that preferentially tags purine bases $(\mathrm{G}$ and $\mathrm{A})$ in nucleic acid polymers. The tagging was confirmed by measuring the average number of bases per molecule of fluorescent dye (approximately 240). For in vitro staining of splenic APCs, the cells were isolated by MACS as described above. Cells were resuspended in FACS buffer and stained at a concentration of 0.1 million cells $/ 50 \mu \mathrm{l}$ with $10 \mu \mathrm{g}$ of tagged dsRNA (pA:pU-F) after a dose-effect optimization study. Following 30 minutes of incubation at $4^{\circ} \mathrm{C}$, the cells were washed and analyzed by flow cytometry (FACSCalibur; Becton, Dickinson and Co.). For competitive inhibition, the cells were preincubated for 10 minutes at $4^{\circ} \mathrm{C}$ with different amounts of nontagged $\mathrm{pA}: \mathrm{pU}, \mathrm{pI}: \mathrm{pC}$, or pA followed by the addition of pA:pU-F as described above.

Isolation of naturally occurring dsRNA. Permissive MDCK cells were infected with WSN influenza virus $\left(10^{8}\right.$ $\mathrm{TCID}_{50} / 1 \times 10^{9}$ cells), and after 24 hours, the cells were harvested and washed, and total RNA was extracted using an RNA separation kit (QIAGEN Inc.). The RNA was further purified by treatment with RNase-free DNase I (Stratagene). The single-stranded RNA (ssRNA) in the samples was then removed by 30 minutes of incubation at $37^{\circ} \mathrm{C}$ with $5 \mathrm{U}$ of S1 nuclease (Ambion Inc., Austin, Texas, USA) per $\mu \mathrm{g}$ RNA. The RNA was analyzed before and after digestion by gel electrophoresis. The absence of infectious properties of the purified dsRNA was confirmed by standard influenza virus titration. As control, we used material similarly treated and purified from $10^{9}$ noninfected MDCK cells. The concentration of nucleic acid was measured by spectrophotometry at an absorbance of $260 \mathrm{~nm}$. The absence of endotoxin was confirmed by Limulus amebocyte lysate assay. The purified dsRNA and control RNA were used individually or as a mixture with recombinant gp140 antigen $(25 \mu \mathrm{g}$ RNA and $2 \mu \mathrm{g}$ antigen in $25 \mu \mathrm{l}$ sterile PBS).

Statistical analysis. Magnitudes of immune responses were compared using the Student $t$ test, assuming a normal distribution of the values and equal variances.

\section{Results}

Systematic definition of RNA motifs that modulate immune responses. During viral infection, transient and unusual RNA species are created and may act as "danger" signals. We postulated that various RNA motifs are being recognized by the innate immune cells and profoundly 
regulate the adaptive immune response. To address this hypothesis on a systematic basis, we screened a library of synthetic ssRNA and dsRNA motifs for the ability to modulate the specific IgG response to a protein antigen (OVA) administered via the respiratory tract. To simplify the process, we organized the screening into two rounds, with round one involving pools of RNA species (Table 1) and the second dissecting components within the pools having maximal impact on the immune response. As shown in Figure 1a, the pool of dsRNA (pool 5) displayed maximal impact on the antibody response, with substantial enhancement of specific immunity. In addition, mixtures of single-stranded species that complement each other (pool 1) partially reproduced this enhancement. This suggests that both the residues in and the secondary structure of an RNA determines its ability to act as a danger motif. In the second round of screening, we show that two categories of motifs organized as dsRNA, namely pA:pU and pI:pC, have a significant impact on the development of IgG response to a specific antigen in C57BL/6 mice (Figure 1b). Furthermore, both pA:pU and pI:pC had an amplifying effect on the specific $T$ cell response (Figure 1c). Surprisingly, the profile of the T cell response was dependent on the nature of the RNA residues, suggesting that the immune system is able to discriminate RNA-associated danger motifs. RNA motifs containing $A$ and $U$ residues rather than $I$ and $C$ residues were able to direct the differentiation of IFN- $\gamma$-producing Th1 cells (Figure 1c) in C57BL/6 mice. This result was reflected in differential induction of IgG isotypes, consistent with the Th profile (Figure 1b, inset). Thus, pA:pU displayed both T1-promoting and antibody-enhancing effects. The stimulatory properties of selected dsRNA motifs on adaptive immunity were preserved in $\mathrm{C} 3 \mathrm{H} / \mathrm{HeJ}$ mice, which have defective TLR-4 and thus are unable to mount a normal response to LPS (Figure 2a).

The impact of dsRNA on antibody response was independently confirmed with additional foreign antigens, namely HIV envelope protein (recombinant gp140) and whole inactivated influenza virus, administered to BALB/c mice (Figure 2, b and c). In fact, pA:pU rather than $\mathrm{pI}: \mathrm{pC}$ restored the titer of specific antibodies to influenza virus in $\mathrm{BALB} / \mathrm{c}$ mice to a level similar to that triggered by infection (Figure $2 c$ ). In addition, in the context of immunization with inactivated virus, $\mathrm{pA}: \mathrm{pU}$ restored the $\mathrm{T}$ cell response to a similar magnitude to that conferred by natural infection (Figure $2 \mathrm{~d}$ ). Thus, different RNA motifs exert a previously unknown and broad range of effects on $\mathrm{T}$ and $\mathrm{B}$ cell responses.

Motifs associated with dsRNA regulate the recruitment and activation of professional APCs. We hypothesized that dsRNA-associated danger motifs such as pA:pU and $\mathrm{pI}: \mathrm{pC}$ influence the $\mathrm{T}$ cell response indirectly via components of innate immunity. To test this hypothesis, we first defined the expression of chemokine genes in the pulmonary lymphoid tissue after administration of RNAs. DNA array technique showed that IP-10, MIG, MIP-1 $\alpha$, MIP-1 $\beta$, and MCP-1 were strongly induced by both pA:pU and pI:pC (Figure 3a). However, only pI:pC triggered expression of RANTES and MCP-3, CC chemokines that have the ability to engage receptors selectively expressed by Th2 cells. LPS induced a different chemokine expression: upregulation of CXC chemokines MIG and MIP- $1 \alpha$ and the CC chemokine TCA-3 (Figure 3a). FACS analysis of pulmonary interstitial cells subsequent to mucosal administration of pA:pU and pI:pC showed prompt recruitment of CD $11 \mathrm{~b}^{+}$monocytes and CD11 $c^{+}$DCs (Figure 3b). There was no substantial
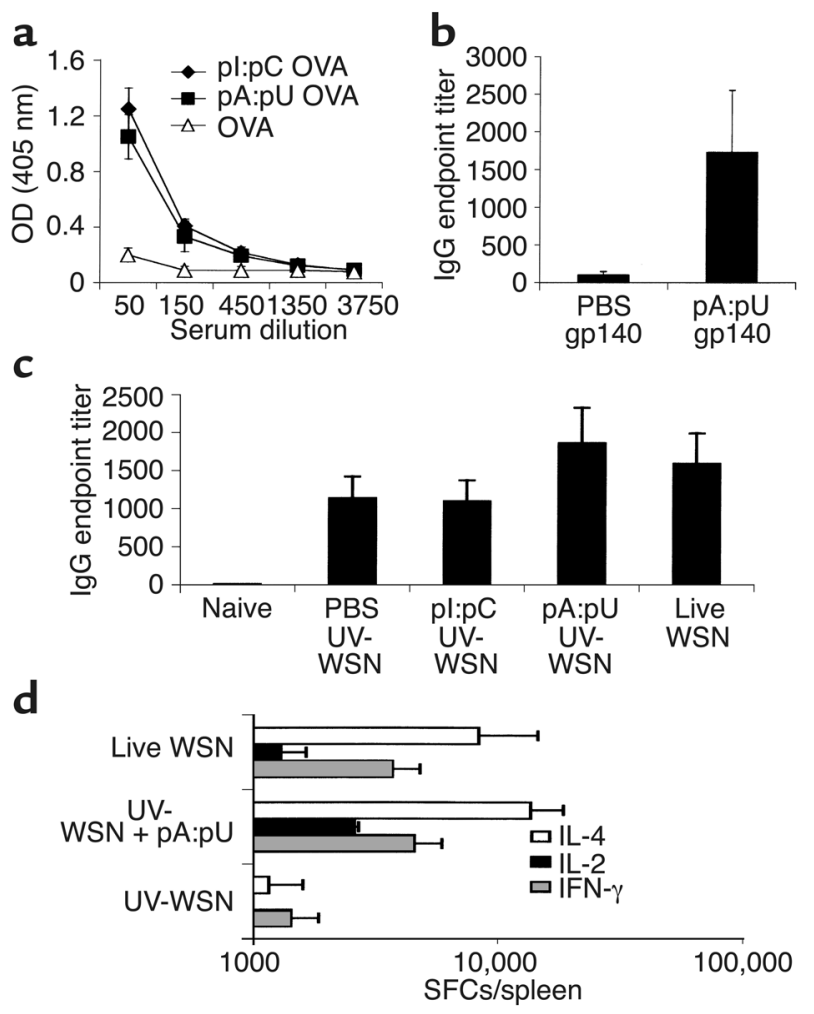

\section{Figure 2}

Increase of immune response to viral antigens by a specific dsRNA motif. (a) The induction of antigen-specific IgG response in $\mathrm{C} 3 \mathrm{H} / \mathrm{HeJ}$ mice deficient in TLR- 4 was measured by ELISA after immunization with OVA together with pl:pC (filled diamonds) or pA:pU (filled squares), or with OVA alone (open triangles). Results are expressed as mean \pm SEM of OD (measured at $405 \mathrm{~nm}$ ) corresponding to different serum dilutions ( $n=3$ mice/group) and are representative of two independent measurements. (b) The effect on the antibody response to the HIV gp140 fragment was measured in female BALB/c mice immunized via the respiratory tract (intratracheal administration followed by two boosts 2 weeks apart, carried out by intranasal instillation) with gp140 alone or gp140 together with pA:pU. The results were expressed as mean \pm SEM of IgG endpoint titers $(n=3$ mice/group). (c) Similarly, influenza virus-specific IgG antibodies were measured after mucosal immunization of female BALB/c mice with UV-inactivated WSN virus (UV-WSN) alone or together with dsRNA motifs. As control we measured the antibody response after infection with the same strain of influenza virus $(n=4 /$ group). (d) The $T$ cell response to whole influenza virus was studied by ELISpot analysis of splenocytes and was expressed as IFN- $\gamma^{+}$(gray bars), $\mathrm{IL}-4^{+}$(white bars), and IL-2+ (black bars) SFCs/spleen (mean \pm SEM, $n=4$ /group) after subtraction of background. The $T$ cell response to antigen together with $\mathrm{pA}: \mathrm{pU}$ was compared with the response to immunization with antigen alone or to influenza virus infection. The experiment was carried out in female BALB/c mice. 


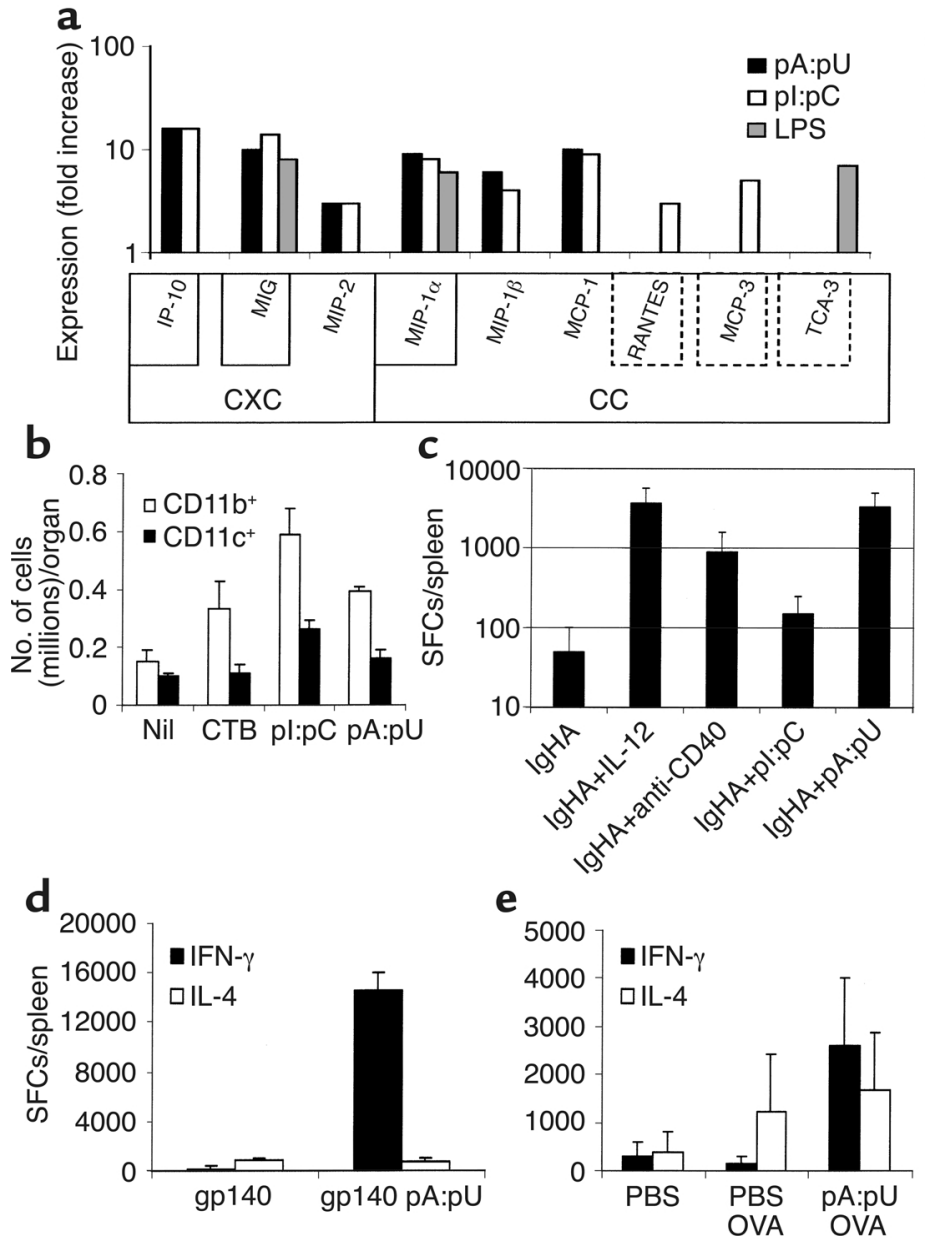

Figure 3

The impact of defined dsRNA motifs on innate immunity and APCs. (a) The chemokine expression triggered by $50 \mu \mathrm{g} \mathrm{pA}: \mathrm{pU}$ (black bars) or pl:pC (white bars) dsRNA was compared with that induced by $1 \mu \mathrm{g}$ of LPS (gray bars) 24 hours after mucosal administration. The chemokines that bind to receptors on Th1 and Th2 cells are indicated with solid or dashed lines. (b) Recruitment of professional APCs in the lungs of mice treated with dsRNA motifs was assessed by FACS. Results are expressed as number of CD11 $\mathrm{c}^{+}$(black bars) and $\mathrm{CD} 11 \mathrm{~b}^{+}$(white bars) cells separated from the pulmonary interstitial tissue ( $n=4 /$ group). (c) Activation of professional APCs by the dsRNA motifs was ascertained by ex vivo overnight pulsing of $C D 11 c^{+}$cells with antigen (100 $\mu \mathrm{g} / \mathrm{ml}$ of $\mathrm{gHA}$ ) together with $50 \mu \mathrm{g} / \mathrm{ml}$ of dsRNA, followed by adoptive transfer of APCs into naive mice. As controls, we used antigen-pulsed APCs or costimulated with either recombinant IL-12 or anti-CD40 mAb. Results are expressed as number of IL-2+ SFCs measured in the spleen. (d) Cross-priming was studied in BALB/c mice immunized with gp140 antigen together or without pA:pU. The response was measured by ELISpot analysis using the peptide $\mathrm{R} 10 \mathrm{~K}$. Results are expressed as mean $\pm \mathrm{SEM}$ of the number of IFN- $\gamma^{+}$(black bars) and IL-4 (white bars) SFCs/spleen ( $n=4$ /group). (e) Cross-priming was studied in C57BL/ 6 mice treated with $100 \mu \mathrm{g}$ of whole OVA together with or without $\mathrm{pA}: \mathrm{pU}$, by ELISpot analysis using in vitro stimulation with the peptide SIINFEKL. Results are expressed in $\mathbf{d}$.

recruitment of leukocytes at the doses studied that may have interfered with the functional capacity of the lungs (25\% increase in the case of $\mathrm{pI}: \mathrm{pC}, 20 \%$ for $\mathrm{pA}: \mathrm{pU}$, and $15 \%$ for CTB, compared with naive mice). In addition, in vitro incubation of $\mathrm{CD} 11 \mathrm{c}^{+} \mathrm{DCs}$ from naive mice with antigen together with pA:pU (and to a lesser extent pI:pC) resulted in their activation, since subsequent adoptive transfer of antigen-pulsed cells into $\mathrm{BALB} / \mathrm{c}$ recipients triggered enhanced class II-restricted T cell immunity (Figure 3c). Similar enhancement has been measured by APC incubation with anti-CD40 antibody or IL-12. Finally, pA:pU had a strong MHC class I-restricted T cell-promoting effect in the context of mucosal vaccination with recombinant HIV envelope protein gp 140 or OVA (Figure 3 , $d$ and e), as revealed by ELISpot analysis with characterized peptides $(16,17)$ from the HIV envelope protein and OVA, respectively. In parallel, we confirmed that negatively separated $\mathrm{CD}^{+}$cells, rather than $\mathrm{CD} 8^{-} \mathrm{T}$ cells, were responsive to the MHC class I-restricted peptide SIINFEKL (data not shown). Thus, dsRNA has the ability to trigger differentiation of professional APCs to a stage compatible with cross-priming of MHC class I-restricted T cells.

Since this process may involve binding to receptors specific for RNA motifs, we tested the binding profile of fluorescently tagged $\mathrm{pA}: \mathrm{pU}$ to APCs in vitro and in vivo. Both $\mathrm{CD} 11 \mathrm{~b}^{+}$and $\mathrm{CD} 11 \mathrm{c}^{+}$ APCs isolated from secondary lymphoid organs or pulmonary tissue displayed subsets that were dimly or intensely stained with pA:pU-F. For example, as shown in Figure 4a, approximately $10 \%$ of splenic CD $11 \mathrm{~b}^{+}$APCs stained intensely when pulsed ex vivo at $4^{\circ} \mathrm{C}$. This staining was effectively inhibited by excess nontagged $\mathrm{pA}: \mathrm{pU}$ and to a lesser extent by $\mathrm{pA}$. In contrast, even a large excess of nontagged pI:pC failed to affect the percentage of APCs highly stained with pA:pU-F. The dimly stained subsets were relatively unaffected in all circumstances, suggesting the coexistence of poorly saturable or nonspecific receptors. When administered via the respiratory tract by intratracheal inoculation in saline, pA:pU-F stained 3-4\% of the local APCs $\left(\mathrm{CD} 11 \mathrm{~b}^{+}\right.$ and $\mathrm{CD} 11 \mathrm{c}^{+}$cells) (Figure $4 \mathrm{~b}$ ). $\mathrm{CD}^{-11 \mathrm{~b}^{-}}$and CD11 c cells were not significantly stained.

To test whether naturally occurring dsRNA promotes the activation of APCs and enhances adaptive immunity, we infected permissive MDCK cells with influenza virus and isolated total RNA after 24 hours. The ssRNA and DNA were removed by treatment with nucleases (see Methods), and the absence of infectious material was confirmed using standard titration on MDCK cells. Incubation of CD11 $c^{+}$DCs with naturally occurring dsRNA or synthetic pA:pU promotes increased production of IL-12 and TNF- $\alpha$ (Figure 4c). No increased production of IL- $1 \beta$ was measured (not shown). In addition, CD11 $\mathrm{c}^{-}$ APCs did not display significant cytokine production. Finally, incubating APCs with control RNA from noninfected MDCK cells did not result in increased cytokine production (Figure 4c). Coadministration of naturally occurring dsRNA with limiting amounts of prototype 
antigen (gp140) to BALB/c mice by the mucosal respiratory route resulted in significant enhancement of the specific IgG response (Figure 4d). Synthetic pA:pU displayed an immune-enhancing effect as well, in contrast to control material from noninfected MDCK cells that failed to promote an enhanced antibody response (Figure $4, \mathrm{~d}$ and e). The effect of naturally occurring dsRNA and of synthetic pA:pU was to promote both T1-controlled IgG2a antibodies and IgG1 antibodies (Figure 4e).

Together, these data show a profound impact of synthetic and naturally occurring RNA motifs on elements of innate immunity that in turn has the ability to regulate the adaptive immune response.

dsRNA motifs block the induction of bigh-zone tolerance and trigger protective antiviral immunity. Danger molecules contribute to discrimination between innocuous antigens and those associated with infectious processes. In high doses, noninfectious purified protein antigens induce unresponsiveness or immunological tolerance. In order to test the danger-signal competence of $\mathrm{pA}: \mathrm{pU}$ and pI:pC motifs, we used the model of tolerance achieved by intravenous inoculation of hIgG. Coinoculation of either pA:pU or $\mathrm{pI}: \mathrm{pC}$ together with $\mathrm{hIgG}$ in saline prevented the induction of $\mathrm{B}$ cell unresponsiveness, as shown by antibody titers after boosting with hIgG in CFA (Figure 5, a and b). The dsRNA-associated motifs could both induce a higher primary response and rescue the secondary response to this prototype antigen. Identical profiles were noted in wild-type, LPS-responsive C57BL/6 mice (Figure 5a), $\mathrm{C} 3 \mathrm{H} / \mathrm{SnJ}$ mice (not shown), and LPS-resistant, TLR-4deficient $\mathrm{C} 3 \mathrm{H} / \mathrm{HeJ}$ mice (Figure $5 \mathrm{~b}$ ). Similarly, assessment of the $\mathrm{T}$ cell profile revealed that IL-4 and IL-2

\footnotetext{
Figure 4

Binding of dsRNA to APCs and the subsequent effect on adaptive immunity. (a) MACS-separated APCs were incubated with $10 \mu \mathrm{g} / \mathrm{ml}$ $\mathrm{pA}: \mathrm{pU}-\mathrm{F}$, then washed and analyzed. Alternatively, APCs were preincubated with 20 or $100 \mu \mathrm{g} / \mathrm{ml}$ of nontagged pA:pU, pA, or pl:pC before staining. The profiles of stained (unshaded region) and nonstained (shaded region) cells and the percentage of highly stained APCs are represented in each panel, with a logarithmic $x$ axis. (b) In vivo binding of pulmonary $\mathrm{CD} 11 \mathrm{c}^{+}$and $\mathrm{CD} 11 \mathrm{~b}^{+} \mathrm{APC}$ by tagged $\mathrm{pA}: \mathrm{pU}$. Animals were treated with $\mathrm{PA}: \mathrm{pU}-\mathrm{F}$ by intratracheal instillation, and lung interstitial cells separated. The cells were stained with PE-tagged isotype control, anti-CD11c, or anti-CD11b antibodies. The percentages of pA:pU$\mathrm{F}$-stained cells were estimated in the gated CD11 $\mathrm{c}^{+}, \mathrm{CD} 11 \mathrm{c}^{-}, \mathrm{CD} 11 \mathrm{~b}^{+}$, and $\mathrm{CD}_{11 b^{-}}$subpopulations. (c) Activation of CD11 $\mathrm{c}^{+} \mathrm{APC}$ by $\mathrm{pA}: \mathrm{pU}$ and viral dsRNA was assessed by measuring the cytokine production. MACS-separated CD11 $c^{+}$or control CD11 $c^{-}$cells were incubated with $25 \mu \mathrm{g} / \mathrm{ml}$ dsRNA, RNA from noninfected cells (ctrl RNA), or no RNA (Ctrl). The cytokines were expressed as mean $\pm \mathrm{SEM}$ in $\mathrm{pg} / \mathrm{ml}$. The right $y$ axis scale is for IL-12 (diamonds) and the left $y$ axis scale is for TNF- $\alpha$ (squares). ( $\mathbf{d}$ and $\mathbf{e}$ ) The effect of dsRNA from influenza virus-infected cells on the response to an antigen (gp140) was measured subsequent to immunization with antigen + dsRNA. The specific $\lg G$ response (d) and the $\operatorname{lgG} 1$ and $\lg \mathrm{g} 2 \mathrm{a}$ components (e) were measured by ELISA. As controls, we used mice immunized with gp140 in PBS, gp140 + pA:pU, or gp140 + ctrl RNA from noninfected cells. Results are expressed as mean $\pm \operatorname{SEM}(n=3$ mice/group) of OD $(405 \mathrm{~nm})$ at various serum dilutions $(\mathbf{d})$ or diluted 100 times $(\mathbf{e})$.
}

production were partially restored by coadministration of dsRNA with hIgG (not shown).

We postulated that danger motifs have the capability to rapidly mobilize protective arms of immune defense. Thus, we tested whether pA:pU and pI:pC have any impact on the evolution of primary infection with influenza virus. Mice treated with RNA motifs via the respiratory tract were infected with sublethal doses of influenza virus. Five days after infection, the pulmonary virus titers were quantified. Concordant results were obtained in C57BL/6 and TLR4-/- $\mathrm{C} 3 \mathrm{H} / \mathrm{HeJ}$ mice (Figure 5, c and d). Notably, pA:pU was more effective than pI:pC in orchestrating an effective reduction of pulmonary virus titers in C57BL/6 mice, and performed similarly in $\mathrm{C} 3 \mathrm{H} / \mathrm{HeJ}$ mice. Thus, in the absence of immune memory, dsRNA motifs are able to mobilize an effective primary response against viral infection, in addition to acting as a "master switch" for adaptive immunity (Figure 5e).

Enhancement of immunity to protein antigens by coencapsulation with RNA danger motifs and mucosal vaccination. Since the immune response to nonformulated subunit vaccines and the general response to purified protein antigens is minimal, we tested whether in vivo coexposure of APCs to antigen and dsRNA danger motifs results in a more favorable outcome. Effective antigen administration to the bronchial lymphoid tissue requires the use of specialized delivery systems. To this aim, we coformulated a prototype antigen (OVA) with pA:pU or pI:pC in vectors

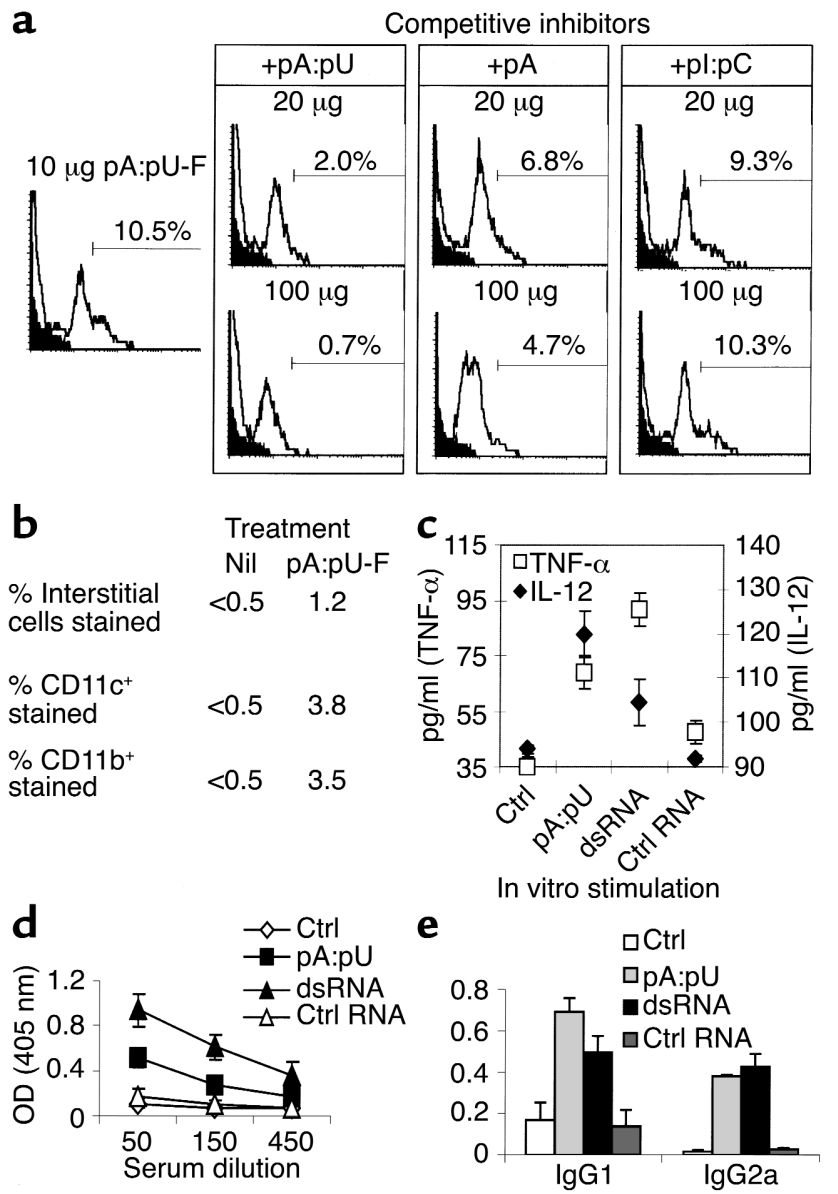




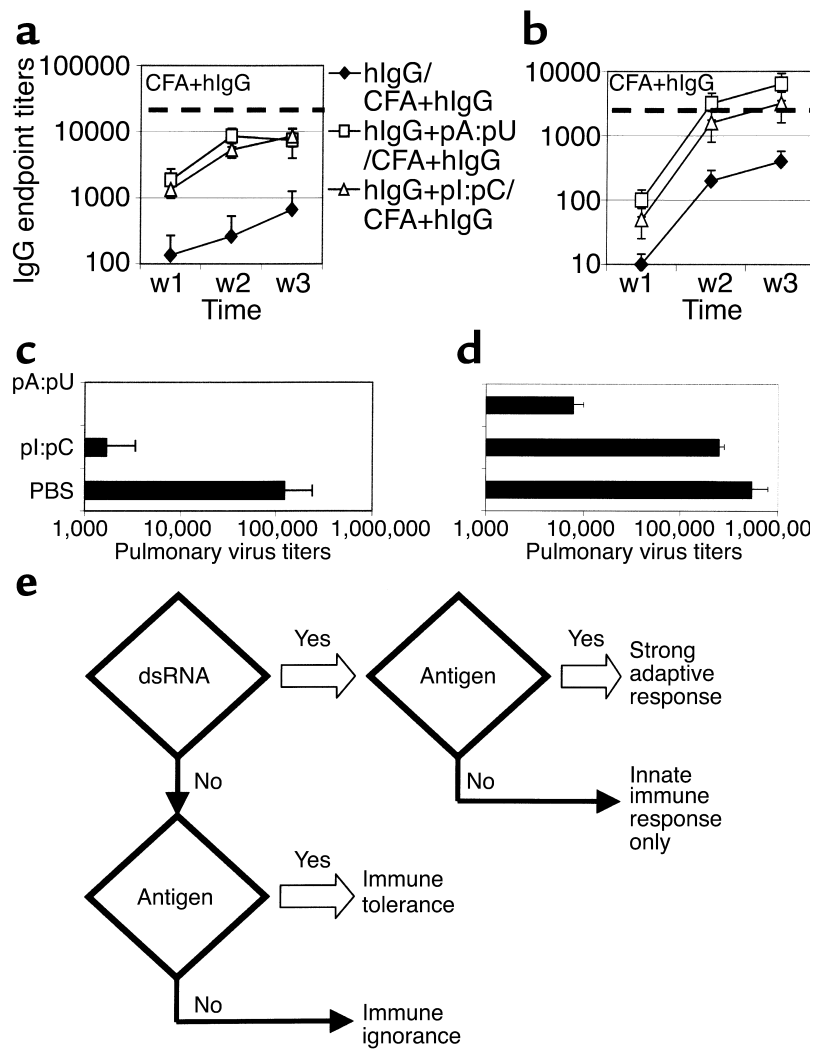

Figure 5

The "danger-signal" quality of dsRNA motifs. ( $\mathbf{a}$ and $\mathbf{b}$ ) dsRNAs prevent high-zone tolerance. C57BL/ 6 mice (a) or LPS-resistant $\mathrm{C} 3 \mathrm{H} / \mathrm{HeJ}$ mice (b) were injected with a tolerogenic dose of hlgG alone or together with pl:pC or pA:pU. Mice were subsequently boosted with an immunogenic dose of hlgG in CFA. The antibodies against hIgG were measured by ELISA at various intervals, as shown in the figure. As control, we included mice immunized with hlgG emulsified in CFA and represented the maximal titer on the graph (dashed line). Results are shown as mean \pm SEM of endpoint titers ( $n=5$ /group). Similar results were obtained in LPS-responsive mice (not shown). (c and $\mathbf{d}$ ) dsRNA motifs display differential ability to mobilize immunity against influenza virus. $\mathrm{C} 57 \mathrm{BL} / 6$ (c) or $\mathrm{C} 3 \mathrm{H} / \mathrm{HeJ}(\mathbf{d})$ mice were treated with pl:pC, pA:pU, or PBS 1 day before and 1 day after pulmonary infection with influenza virus. On day 5 , the virus titer in the pulmonary tissue was estimated and represented as $\mathrm{TCID}_{50}$ /organ (mean $\pm \mathrm{SEM}$, $n=6 /$ group). Results are representative of studies in $T L R 4^{-/-}$and competent mice. (e) Schematic depiction of the involvement of dsRNA danger motifs in the regulation of immunity: detection of dsRNA motifs triggers a "master switch" for B and T cell responses to coexisting antigens. In the absence of danger signals, the magnitude of immunity is low if the antigen is limiting (immune ignorance), or immune tolerance develops if antigen is abundant.

(SC-Vs) that can be easily delivered as aerosols and are composed of biocompatible excipients such as phospholipids and lactose. After delivery of either OVA plus either pA:pU or $\mathrm{pI}: \mathrm{pC}$ formulated in SC-V to the respiratory tract of C57BL/6 mice, we measured antibody responses that were considerably higher than those of mice immunized with nonformulated antigen in PBS or with antigen formulated in vectors devoid of dsRNA motifs (Figure 6a). Interestingly, CTB had only a limited adjuvant effect in the context of SC-V coformulation. Consistent with previous results, induction of $\mathrm{T} 1$ immunity was measured only with pA:pU particles (Figure 6b) and not with pI:pC, which displayed only enhancement of T2 immunity (not shown). Furthermore, the $\mathrm{T}$ cell response to pA:pU coformulated antigen displayed an important local component (Figure 6b). Finally, using the welldefined class I-restricted SIINFEKL peptide, we confirmed that $\mathrm{pA}: \mathrm{pU}$ retains the ability to promote crosspriming in conjunction with the vectors used (Figure 6b).

A similar enhancement of the antibody response has been measured in Sprague-Dawley rats aerosolized with vectors loaded with OVA together with $\mathrm{pA}: \mathrm{pU}$ or $\mathrm{pI}: \mathrm{pC}$ (Figure 6c). Lower titers were achieved with SC-V devoid of dsRNAs or OVA in saline. The analysis of mucosal antibody titers (Figure 6d) revealed a similar profile.

Thus, coformulation of RNA-associated danger motifs and protein antigen using this formulation technology results in a substantial increase in the specific immune response with potential clinical implications.

\section{Discussion}

The mechanisms of immune response during microbial infection became a major area of investigation subsequent to advances in understanding the potential role of innate immunity. It quickly became evident that the innate immune cells are endowed with multiple categories of receptors that discriminate between various microbe-associated motifs or exogenous danger signals. Crosstalk between the innate and adaptive immune systems subsequent to such pattern recognition events decisively influences the magnitude and profile of $\mathrm{T}$ and $\mathrm{B}$ cell responses. The innate immune system is rapid though less discriminative, but instructs the adaptive immunity that evolves more slowly and is composed of more efficient effectors with a vast repertoire acquired by somatic mutation. This multipatterned recognition strategy that jointly employs innate and adaptive immunity shifted the immune discrimination paradigm from self/nonself to dangerous/nondangerous cognition. The poor immunogenicity of purified proteins, induction of immune mediators by microbial motifs, and characterization of the activity of such mediators (cytokines, chemokines, and costimulatory molecules) on adaptive immunity all supported this concept (18). In the present study, we undertook a rational approach in delineating the role of noncoding RNA motifs as danger signals, with direct implications for understanding their role in controlling adaptive immunity during viral infections. In addition, we explored their use as adjuvants in conjunction with mucosal vaccination.

We employed a library of synthetic RNAs and a twotier strategy, using as read-out the effect on adaptive rather than innate immunity. By this method, we found, surprisingly, that the oligonucleotide composition in addition to the nature of RNA as double-stranded or single-stranded plays a role in this concern. We show that AU-based motifs have the ability to turn on Th1 immunity, isotype switching to IgG2a (Figure 1), and cross-priming (Figure 3 ) to a greater extent than 
IC- or CG-based motifs do. IC motifs defined earlier (19) resulted in enhanced T2 and B cell immunity in a manner dependent on the antigen and the animal strain (Figure 1 and Figure 2). CG motifs associated with dsRNA, and miscellaneous ssRNA motifs, had litthe effect on adaptive immunity unless mixtures of ssRNAs composed of complementary bases were used. Since these findings have been reproduced in the absence of functional TLR-4 (Figure 2 and Figure 5), a pathway common to that of endotoxin recognition can be ruled out at this point. Recently, it has been shown that TLR-3 plays a role in recognition of $\mathrm{pI}: \mathrm{pC}$ (7), but a common recognition pathway with $\mathrm{pA}: \mathrm{pU}$ seems unlikely due to the profoundly different profile of the immune response triggered by these two motifs (Figure 1). In addition, there was a clear-cut difference in the ability of pA:pU and pI:pC to antagonize the binding of tagged $\mathrm{pA}: \mathrm{pU}$ to APC receptors (Figure 4), strongly supporting a model based on multiple receptors for RNA motifs. Thus, different TLR isoforms and/or coreceptors may participate in the process of discriminating RNA motifs based on the identity of nucleotides, in a process reminiscent of a rudimentary immune repertoire. In support of this concept, pA showed binding with reduced affinity to the pA:pU receptor on APCs (Figure 4). Thus, nucleotide composition and the single- versus double-stranded nature of the RNA may affect its secondary and tertiary structure, resulting in differential binding affinity to the same receptor or various isoforms. A possibility may be that TLR-9, shown to recognize palindromic unmethylated $\mathrm{CpG}$ oligodeoxynucleotide motifs (20), or isoforms of TLR, may be involved in dsRNA motif discrimination. Recent evidence does not support the involvement of TLR-9, since dsRNA induces a different spectrum of transcription factors and costimulatory molecules than unmethylated $\mathrm{CPG}$ motifs induce (21). Since both $\mathrm{pI}: \mathrm{pC}$ and $\mathrm{pA}: \mathrm{pU}$ induce CXC chemokines (Figure 3), it is likely that alternative mediators such as CC chemokines with the ability to selectively bind Th2 cells (22) may be responsible for the different $\mathrm{Th}$ profiles elicited by these motifs. In addition, this may explain the distinct effect of pI:pC and $\mathrm{pA}: \mathrm{pU}$ relative to the type of APCs recruited at the site of administration (Figure 3).

Based on the data, we conclude that the newly characterized pA:pU-associated motifs can induce a large number of features of the adaptive immune response that are usually noted only after viral infection. Induction of $\mathrm{T} 1$ responses (both Th1

\section{Figure 6}

and $\mathrm{Tc} 1$ ) was documented with protein antigens (OVA and gp140) and inactivated influenza virus (Figures 1-3). Induction of an MHC class I-restricted response to protein antigens suggests that this RNA motif activated APCs to a level compatible with this mechanism of processing and presentation, concordant with observations implying cross-priming in viral infections (23). This suggests that RNA-associated danger motifs rather than direct infection of APCs may be responsible for induction of cytotoxic $T$ lymphocytes during infection with RNA viruses such as influenza virus. The enhanced magnitude of the immune response can be explained by rapid recruitment and activation (Figure 3) of APCs. Despite the fact that at this point, the differential activity of pA:pU and pI:pC cannot be ascribed to any single cytokine or chemokine, a more detailed analysis of the profile of activated APCs is likely to
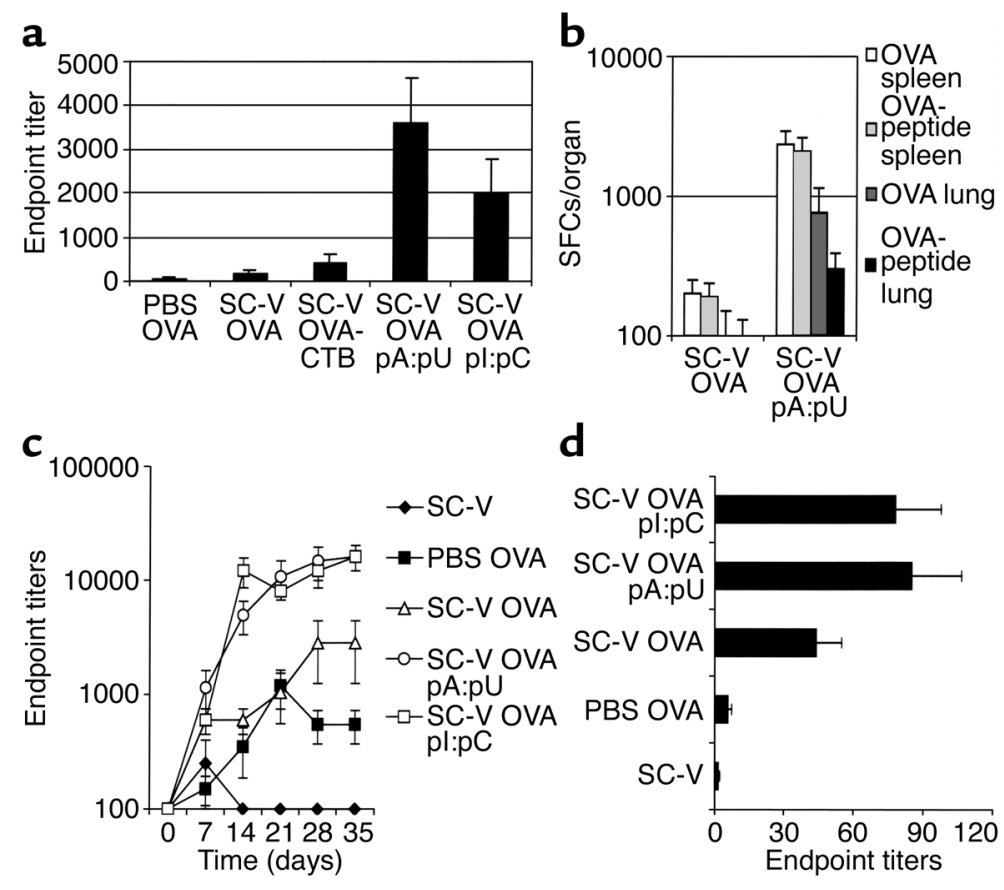

Use of selected dsRNA motifs as potent vaccine adjuvants in a context of engineered vectors. (a) SC-Vs loaded with model antigen (OVA) alone or together with dsRNA motifs were generated and tested first in C57BL/ 6 mice. The antibody response was measured by ELISA and shown as mean $\pm \operatorname{SEM~(~} n=4$ /group; data are representative of two independent measurements) of IgG endpoint titers at 2 weeks after intratracheal immunization. As controls, we used OVA in PBS and OVA coformulated with CTB in SC-V. (b) Local (lung) and systemic (splenic) T cell response in C57BL/6 mice to whole OVA antigen or class I-restricted dominant OVA peptide measured in mice immunized with OVA encapsulated in SC-V with or without pA:pU. The analysis was carried out by ELISpot and the results are expressed as IFN- $\gamma^{+}$SFCs per organ (mean \pm SEM; $n=4$ /group). (c) Systemic and (d) local antibody response of Sprague-Dawley rats to mucosal vaccination with SC-V loaded with model antigen (OVA) coformulated with dsRNA motifs. The dose of antigen per rat was $100 \mu \mathrm{g}$ OVA with or without $50 \mu \mathrm{g}$ pl:pC or pA:pU dsRNA. The material was aerosolized into the trachea of anesthetized rats. As controls, we used SC-V loaded with OVA but devoid of dsRNA motifs (open triangles), dose-matched amounts of OVA in PBS (filled squares), and SC-V devoid of antigen (filled diamonds), respectively. The results are expressed as endpoint titers (mean \pm SEM; $n=4$ /group) of OVA-specific IgG antibodies measured by ELISA in serum (c) and in bronchoalveolar lavage fluid on day 35 (d). 
address this issue. The induction of $\mathrm{T} 1 \mathrm{immunity}$ promoted by pA:pU was accompanied by isotype switching, resulting in generation of $\operatorname{IgG} 2 \mathrm{a}$ antibodies. However, dsRNA could not induce an isotype switch to the IgA class. This was associated with inhibition of TGF- $\beta$ (not shown), suggesting that dsRNA danger motifs act by virtue of induction of proinflammatory mediators and downregulation of anti-inflammatory mediators. These results are consistent with a considerable, but not exclusive, role of dsRNA motifs in shaping adaptive immunity during infections such as influenza, as depicted in the Figure 5e.

Since potent danger motifs should influence the outcome in terms of immune responsiveness versus tolerance, we studied whether dsRNAs prevent highzone tolerance to hIgG, a well-characterized model of immune unresponsiveness (24). We show that both $\mathrm{pA}: \mathrm{pU}$ and $\mathrm{pI}: \mathrm{pC}$ are potent inhibitors of immune tolerance (Figure 5, a and b), not only modulators of the adaptive response. This observation enriches the panel of features borne by dsRNAs and opens up the possibility that adjuvant effects of danger motifs in general are caused at least in part by prevention of immune unresponsiveness. Our data are concordant with a recent report showing that $\mathrm{pI}$ : $\mathrm{pC}$ promoted loss of tolerance to a defined autoantigen (25). Lastly, since bases $\mathrm{A}$ and $\mathrm{U}$, but not $\mathrm{I}$, are found in natural RNA species, our data point to the first dsRNA motif with potential relevance to the immune response during viral infections. This concept is being strongly supported by the finding that naturally occurring dsRNA from virus-infected cells stimulated DCs and promoted increased specific responses to coadministered protein antigen (Figure 4).

The potency of $\mathrm{pA}: \mathrm{pU}$ as a danger motif has been illustrated by its ability to control primary infection with influenza virus (Figure 5, c and d). This feature, which can be explained by rapid mobilization of innate and adaptive responses, is highly reminiscent of the ability of unmethylated CPG oligoDNA motifs to improve on immune defense during primary infection (26). By extrapolation, we conclude that the innate immune system has an exquisite ability to recognize foreign or endogenously generated infection-associated polynucleotide motifs and to accordingly regulate adaptive immunity.

Based on the results of this study, dsRNA motifs appear to be logical candidates for adjuvants in conjunction with subunit, recombinant, or inactivated vaccines. In particular, pA:pU appears likely to provide some of the beneficial features of live vaccines in the absence of vector replication. This concept was supported by our results on biocompatible aerosols as delivery vectors designed for mucosal vaccination (Figure 6).

In conclusion, by using a rational approach of selecting RNA motifs that impact the adaptive immune responses, we defined an unexpected heterogeneity of RNA-associated danger motifs. A systematic study of the adaptive immune response demonstrated that selected RNA motifs orchestrate a broad range of features that are reminiscent of natural infection. Finally, this study defines a practical approach consisting of novel formulations containing such RNA motifs that are of potential use in mucosal vaccination.

\section{Acknowledgments}

This work was supported by Alliance Pharmaceutical Corp. and by NIH grant 1R21 AI-47014 (to A. Bot).

1.Zinkernagel, R.M., and Doherty, P.C. 1997. The discovery of MHC restriction. Immunol. Today. 18:14-17.

2. Weigle, W.O. 1987. Factors and events in the activation, proliferation, and differentiation of B cells. Crit. Rev. Immunol. 7:285-324.

3. Matzinger, P. 2001. Introduction to the series. Danger model of immunity. Scand. J. Immunol. 54:2-3.

4. Kruisbeek, A.M., and Amsen, D. 1996. Mechanisms underlying T-cell tolerance. Curr. Opin. Immunol. 8:233-244.

5. Miller, J.F. 1993. Self-nonself discrimination and tolerance in T and B lymphocytes. Immunol. Res. 12:115-130.

6. Mellits, K.H., Kostura, M., and Mathews, M.B. 1990. Interaction of adenovirus VA RNAl with the protein kinase DAI: nonequivalence of binding and function. Cell. 61:843-852.

7. Alexopoulou, L., Holt, A.C., Medzhitov, R., and Flavell, R.A. 2001. Recognition of double-stranded RNA and activation of NF-kappaB by Tolllike receptor 3. Nature. 413:732-738.

8. Braganca, J., and Civas, A. 1998. Type I interferon gene expression: differential expression of IFN-A genes induced by viruses and doublestranded RNA. Biochimie. 80:673-687.

9. Cella, M., et al. 1999. Maturation, activation, and protection of dendritic cells induced by double-stranded RNA. J. Exp. Med. 189:821-829.

10. Le Bon, A., et al. 2001. Type I interferons potently enhance humoral immunity and can promote isotype switching by stimulating dendritic cells in vivo. Immunity. 14:461-470.

11. Haines, D.S., Strauss, K.I., and Gillespie, D.H. 1991. Cellular response to double-stranded RNA. J. Cell. Biochem. 46:9-20.

12. Bhardwaj, N., et al. 1994. Influenza virus-infected dendritic cells stimulate strong proliferative and cytolytic responses from human $\mathrm{CD}^{+} \mathrm{T}$ cells. J. Clin. Invest. 94:797-807.

13. Earl, P.L., et al. 2001. Immunogenicity and protective efficacy of oligomeric human immunodeficiency virus type 1 gp140. J. Virol. 75:645-653.

14. Zaghouani, H., et al. 1993. Presentation of a viral T cell epitope expressed in the CDR3 region of a self immunoglobulin molecule. Science. 259:224-227.

15. Isobe, H., Alt, F., Bona, C.A., and Schulman, J. 1996. Intact antiinfluenza virus immune response in targeted kappa-deficient mice. Viral Immunol. 7:25-29.

16. Nehete, P.N., Casement, K.S., Arlinghaus, R.B., and Sastry, K.J. 1995. Studies on in vivo induction of HIV-1 envelope-specific cytotoxic T lymphocytes by synthetic peptides from the V3 loop region of HIV-1 IIIB gP 120. Cell. Immunol. 160:217-223.

17. Stura, E.A., et al. 1992. Crystallization of murine major histocompatibility complex class I H-2K $\mathrm{K}^{\mathrm{b}}$ with single peptides. J. Mol. Biol. 228:975-982.

18. Gallucci, S., and Matzinger, P. 2001. Danger signals: SOS to the immune system. Curr. Opin. Immunol. 13:114-119.

19. Absher, M., and Stinebring, W.R. 1969. Toxic properties of a synthetic double-stranded RNA. Endotoxin-like properties of poly I. poly C, an interferon stimulator. Nature. 223:715-717.

20. Hemmi, H., et al. 2000. A Toll-like receptor recognizes bacterial DNA. Nature. 408:740-745.

21. Suzuki, K., et al. 1999. Activation of target-tissue immune-recognition molecules by double-stranded polynucleotides. Proc. Natl. Acad. Sci. USA. 96:2285-2290.

22. Ward, S., Bacon, K., and Westwick, J. 1998. Chemokines and T lymphocytes: more than an attraction. Immunity. 9:1-11.

23. Prasad, S.A., et al. 2001. Cutting edge: recombinant adenoviruses induce $\mathrm{CD} 8 \mathrm{~T}$ cell responses to an inserted protein whose expression is limited to nonimmune cells. J. Immunol. 166:4809-4812.

24. Nelson-Rampy, P.A., Parks, D.E., and Weigle, W.O. 1981. Establishment of unresponsiveness in primed B lymphocytes in vivo. J. Immunol. 127:1415-1419.

25. Moriyama, H., et al. 2002. Induction and acceleration of insulitis/diabetes in mice with a viral mimic (polyinosinic-polycytidylic acid) and an insulin self-peptide. Proc. Natl. Acad. Sci. USA. 99:5539-5544.

26. Klinman, D.M., Verthelyi, D., Takeshita, F., and Ishii, K.J. 1999. Immune recognition of foreign DNA: a cure for bioterrorism? Immunity. 11:123-129. 